\title{
Can Tao-Eldrup Model Be Used at Short o-Ps Lifetime?
}

\author{
B. ZGARDZIŃSKA*, \\ Department of Nuclear Method, Institute of Physics, M. Curie-Skłodowska University \\ pl. M. Curie-Skłodowskiej 1, 20-031 Lublin, Poland
}

\begin{abstract}
The distortion of estimated void radii introduced by assumption of an infinite potential depth in the TaoEldrup model is discussed. If the deviation by $10 \%$ is still acceptable, the range of ortho-Ps lifetimes at which the model gives correct radii is from 1.8 to $6.2 \mathrm{~ns}$.
\end{abstract}

DOI: $10.12693 /$ APhysPolA.125.700

PACS: $78.70 . \mathrm{Bj}, 14.60 . \mathrm{Cd}$

\section{The model}

The most popular model describing the relation between ortho-positronium (o-Ps) lifetime and the size of free volume in which it is located was proposed by Tao [1] and modified by Eldrup [2]. The empty void represents for positronium a potential well and the decay constant $\lambda_{3}$ of $o$-Ps in the well is the product of probability $P$ to find $o$-Ps outside the well and the decay constant $\lambda_{\mathrm{b}}$ of $o$-Ps in the bulk

$$
\lambda_{3}=P \lambda_{\mathrm{b}} \text {. }
$$

Due to short range of atomic interactions, the shape of potential is usually assumed stepwise (rectangular) and the geometry is spherical. Thus,

$$
P=4 \pi \int_{R}^{\infty} \psi_{\text {out }}^{2}(r) r^{2} \mathrm{~d} r
$$

where $R$ - the well radius. The radial wavefunction $\psi_{\text {out }}(r)$ of structureless positronium outside the well is of $A \exp (-\kappa r) / r$ type. The constants $A$ and $\kappa$ depend on the well radius, the depth of the well $U$, and also, as stressed recently by Stepanov [3], on the dielectric constant of the medium; $\lambda_{b}$ should also depend e.g. on medium electron density.

To simplify the calculations of the integral (2) Tao proposed to substitute the finite well by an infinite one, and to shift the potential step from $R$ to $R+\Delta$, where the parameter $\Delta$ fulfills the condition

$$
\int_{R}^{\infty} \psi_{\text {out }}^{2}(r) r^{2} \mathrm{~d} r=\int_{R}^{R+\Delta} \psi_{\text {in }}^{2}(r) r^{2} \mathrm{~d} r,
$$

where $\psi_{\text {in }}$ is the wave function for a particle inside the well; $\psi_{\text {in }} \sim \sin k r / r$. Moreover, the decay constant in the bulk is assumed density independent; it is equal to the weighted average of the decay constants of ortho-Ps and para-Ps in vacuum, $2 \mathrm{~ns}^{-1}$. In such a model

$$
\lambda_{3}=\lambda_{\mathrm{b}}\left(1-\frac{R}{R+\Delta}+\frac{1}{2 \pi} \sin \frac{2 \pi R}{R+\Delta}\right) .
$$

If the void radii are known from an independent source

\footnotetext{
*e-mail: bozena.zgardzinska@poczta.umcs.lublin.pl
}

and the values of $\lambda_{3}$ from positron annihilation lifetime spectra, one can find $\Delta$ as an empirical constant. In a series of papers by Eldrup [2,4-7] the Ps traps in plastic crystals were identified as vacancies, thus their sizes could be determined from the crystallographic data (as the Wigner-Seitz radii of molecules). On the other hand, the $o$-Ps lifetimes were measured, lying in the range from $2.45 \mathrm{~ns}$ in succinonitril [4] to $3.2 \mathrm{~ns}$ in camphene [4]. The best agreement between the radii determined from Eq. (4) and the Wigner-Seitz radii was obtained for $\Delta=0.17 \mathrm{~nm}$ (now the commonly accepted $\Delta$ value is $0.166 \mathrm{~nm})$.

The Tao-Eldrup (TE) model contains many approximations. They are well justified for the range of lifetimes as written above, but it needs evaluation how accurate the model is for the radii far from the range appearing in plastic crystals. It seems that the most important source of possible deviations is assumption of infinitely deep potential well. Positronium in a void can exist if there are the energy levels for such particle (at least one level). In the well infinitely deep, the energy levels always exist for an arbitrarily narrow radius. At a limited depth, there is the minimal radius $R_{\min }$, at which there is the level (near the upper rim of the well):

$$
R_{\min }=\frac{0.216 \mathrm{~nm}}{\sqrt{U}},
$$

for $U$ in eV. It seemed worthwhile to check how far the radii found from TE model deviate from these determined using the realistic depth of potential well.

\section{Results and discussion}

The radii of vacancies from Eldrup's works lie in the range $0.32-0.38 \mathrm{~nm}$, thus let us assume that in the middle of that range, $0.35 \mathrm{~nm}$, Eq. (4) gives correct value of the $o$-Ps lifetime. At finite depth of well the probability $P$ (Eq. (2)) depends on that depth, $U$. There are no direct data what values of $U$ appear in the case of organic compounds. In the literature one can find the values close to $1 \mathrm{eV}$, determined for liquids (bubble model, [8]), but they can contain the systematic error, as they were obtained assuming the macroscopic value of surface tension. In the case of solids, the time of flight method [9] allows to 
estimate $U$, its values lie in the range $(1 \div 3) \mathrm{eV}$. Calculations of $P$ (and then the lifetime) in this paper were performed as described by Nakanishi and Jean [8]. If at $R=0.35 \mathrm{~nm}$ the TE model is correct, identical $P$ value in the rectangular potential of depth $U$ needs to assume $U=1.50 \mathrm{eV}$. This value was used in our calculations of $R$ vs. $\lambda_{3}$ dependence. The result is shown in Fig. 1. To show the sensitivity of results to the value of potential depth, the calculations were repeated assuming that full agreement of TE model and the version with finite potential depth appears at the radius $R=0.40 \mathrm{~nm}$. At this assumption the value of $U$ should be $1.39 \mathrm{eV}$. The respective curve is also shown in Fig. 1.

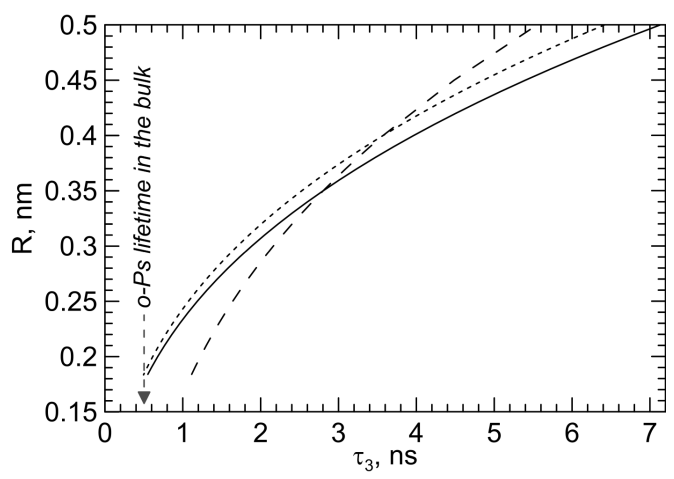

Fig. 1. Relation between the ortho-positronium lifetime and void radius. Tao-Eldrup model (dashed line), finite potential well depth: $1.50 \mathrm{eV}$ (solid line) and $1.39 \mathrm{eV}$ (dotted line).

From the practical viewpoint it is important to know the range of lifetimes at which the deviation of TaoEldrup model from the realistic finite potential depth calculations does not exceed certain limits. Both versions assume the same approximations: ideal spherical shape of the void, stepwise change of potential, identity of the location of density step and of potential step for positronium etc., thus with so many approximations, the deviation by $10 \%$ seems still acceptable. Figure 2 shows the relative difference of $R$ calculated from TE model and at finite potential depth. If we tolerate the inconsistency below $10 \%$, the TE model can be used safely at the observed $o$-Ps lifetimes from $1.8 \mathrm{~ns}$ to about $6.2 \mathrm{~ns}$. Limiting the acceptable deviation to $5 \%$ we obtain the range of lifetimes from $2.2 \mathrm{~ns}$ to $3.7 \mathrm{~ns}$ only. At shorter lifetimes the distortion introduced by the use of infinitely deep potential rises rapidly and at $1.2 \mathrm{~ns}$ it amounts to $30 \%$. The numbers given above relate to the potential $1.50 \mathrm{eV}$. In organic solids, in particular at low temperatures, the lifetimes very often lie in the range $1.0 \div 1.4 \mathrm{~ns}$ and application of TE model gives the void sizes evidently too small.

The intention of this note is not to propose the final solution of the problem how to estimate the cavity radius from the Ps lifetime data, but an appeal to be cautious when applying the model which is commonly used. In the discussion presented here we put aside the question of $\lambda_{\mathrm{b}}$ value, which is assumed independent of electron density. However, if we limit our interest to the organic media of similar density, similar elementary composition (mainly carbon and hydrogen, possible small addition of oxygen or nitrogen) the value of bulk $o$-Ps lifetime can be treated as a constant. It need not to be true for the media based on silica, zeolites, etc.

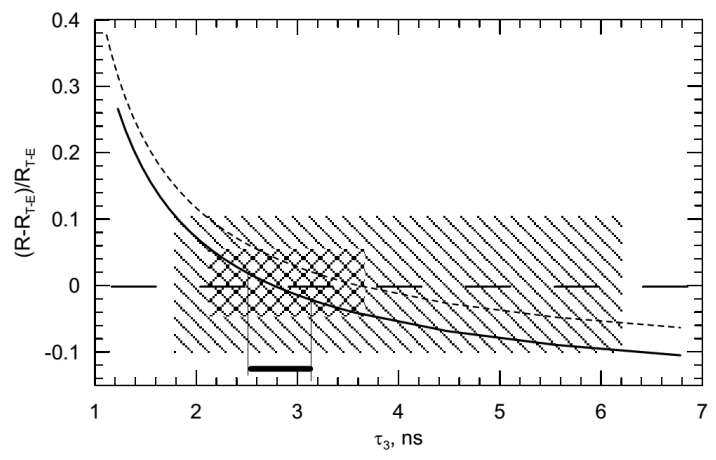

Fig. 2. Relative difference of radii $R$ calculated from TE model and at finite potential depth $U=1.50 \mathrm{eV}$ (solid line) and $1.39 \mathrm{eV}$ (dotted line). Dashed area represents deviation $<10 \%$, checkered area $<5 \%$. The range of $o$-Ps lifetimes from Eldrup papers is shown by the bar above the abscissa axis.

As to the long $o$-Ps lifetimes, one has to remember that this simplest version of the Tao-Eldrup model cannot be used for void radii over $2 \mathrm{~nm}$, when it has to be taken into account the $o$-Ps annihilation not only from the lowest state of Ps particle in the potential well, but also from the excited ones [10].

\section{References}

[1] S.J. Tao, J. Chem. Phys. 56, 5499 (1972).

[2] M. Eldrup, D. Lightbody, J.N. Sherwood, Chem. Phys. 63, 51 (1981).

[3] S.V. Stepanov, D.S. Zvezhinskiy, V.M. Byakov, Mater. Sci. Forum 733, 7 (2013).

[4] M. Eldrup, N.J. Pedersen, J.N. Sherwood, Phys. Rev. Lett. 43, 1407 (1979).

[5] D. Lightbody, M. Eldrup, J.N. Sherwood, Chem. Phys. Lett. 70, 487 (1980).

[6] D. Lightbody, J.N. Sherwood, M. Eldrup, Mol. Cryst. Liq. Cryst. 96, 197 (1983).

[7] D. Lightbody, J.N. Sherwood, M. Eldrup, Chem. Phys. 94, 475 (1985).

[8] H. Nakanishi, Y.C. Jean, in: Positron and Positronium Chemistry, Eds. D.M. Schrader, Y.C. Jean, Elsevier, Amsterdam 1988, p. 169.

[9] Y. Nagashima, Y. Morinaka, T. Kurihara, Y. Nagai, T. Hyodo, T. Shidara, K. Nakahara, Phys. Rev. B 58, 12676 (1998).

[10] T. Goworek, K. Ciesielski, B. Jasińska, J. Wawryszczuk, Chem. Phys. 230, 305 (1998). 